\title{
Mechanical recanalization of acute carotid terminus occlusion from traumatic arterial dissection
}

\section{Michael Chen*}

Department of Neurological Sciences, Rush University Medical Center, Chicago, IL, USA

\section{Edited by:}

Osama O. Zaidat, Medical College of Wisconsin and Froedtert Hospital, USA

\section{Reviewed by:}

Osama O. Zaidat, Medical College of Wisconsin and Froedtert Hospital, USA

Aamir Badruddin, Medical College of

Wisconsin, USA

Thanh Nguyen, Boston Medical Center, USA

Jose R. Romero, Boston University, USA

\section{*Correspondence:}

Michael Chen, Department of

Neurological Sciences, Rush University

Medical Center, 1725 West Harrison

Street, Suite 1121, Chicago, IL 60612,

USA.

e-mail:michael_chen@rush.edu
In the setting of an acute stroke caused by arterial dissection, navigating a microcatheter and microguidewire past the dissected artery to gain access to the distal thromboembolic lesion may exacerbate the underlying pathology. We review a case report whereby successful recanalization of an acute carotid terminus occlusion due to intimal-media dissection in the cervical carotid artery emphasizing the importance of aggressive proximal guide catheter aspiration in conjunction with flow arrest from the carotid bulb proximal to the dissection. We discuss the strengths and limitations of this approach and underscore the importance of a pathology-based approach to acute stroke therapy.

Keywords: acute stroke, carotid terminus occlusion, aspiration, arterial dissection

\section{INTRODUCTION}

Arterial dissection is the second leading cause of stroke in patients younger than age 45 (Lisovoski and Rousseaux, 1991). Current endovascular recanalization approaches for acute stroke focus on either removal of the distal clot using either a retrieval device or aspiration, or displacement via angioplasty and/or self-expanding stent. This approach is problematic in the setting of cervical carotid or vertebral dissection because microcatheter and microguidewire navigation across the dissection has the potential to enlarge the false lumen. We present a case of an acute carotid terminus occlusion caused by clot from a cervical carotid dissection initially worsened by attempts at gaining microcatheter access to the distal clot. However, with proximal flow arrest and primary aggressive proximal guide catheter aspiration, we were able to successfully recanalize the internal carotid artery and its branches, leading to a better than expected clinical outcome.

\section{CASE REPORT}

A 34-year-old man was driving a car when he was hit by another car causing the vehicle to spin completely around. He walked away from the accident with neck discomfort but no neurologic deficits. A week later, he developed sudden onset complete left arm weakness and global aphasia. Intravenous thrombolytics were given, and he was transferred to our hospital with a National Institute of Health Stroke Scale score of 16

Initial cervical carotid catheter angiography revealed a right posterior cervical carotid intimal flap indicating an intimal-media arterial dissection (Figure 1). Cerebral views showed delayed anterograde filling in the internal carotid artery associated with a carotid terminus occlusion (Figure 2). Attempts at advancing a Penumbra 41 reperfusion catheter (Penumbra Inc, Alameda, CA, USA) intracranially were unsuccessful because the edge of the reperfusion catheter could not be advanced past the dissec- tion flap at the distal cervical carotid artery (Figure 3). Subsequent angiography showed a more proximal occlusive lesion, suggesting that the intimal flap had extended into the petrous carotid with further compromise of the luminal diameter (Figure 4).

Using a Merci $8 \mathrm{~F}$ balloon guide catheter (Concentric Medical, Mountain View, CA, USA), a Merci 18L (Concentric Medical, Mountain View, CA, USA) microcatheter was advanced over a microguidewire into the M1 segment. Using standard technique, with a pull lasting 2-3 min, an L6 Merci retrieval device (Concentric Medical, Mountain View, CA, USA) retrieved some clot but only TIMI 1 anterograde flow was achieved. The microcatheter was again advanced up into the M1 segment, a L5 Merci retriever (Concentric Medical, Mountain View, CA, USA) deployed, the balloon inflated, and this time, more aggressive proximal aspiration was performed using a 60-cc syringe. The retriever was visualized under fluoroscopy not to deform its shape during the entire withdrawal, which again lasted only about $1 \mathrm{~min}$, suggesting little or no clot was engaged or retrieved. No clot was visualized on the retriever upon removal. Subsequent angiographic runs, however, demonstrated TIMI 3 recanalization of the internal carotid artery with a persisting but non-occlusive intimal flap along the petrous/cervical section (Figure 5).

Once flow was restored, we decided against placing a stent into the cervical and petrous sections of the carotid artery. The patient had already received $0.9 \mathrm{mg} / \mathrm{kg}$ of intravenous t-PA hours before. We felt that he would have an unacceptably high risk of hemorrhagic transformation by adding the loading dose of abciximab as a prerequisite for stent placement. Anticoagulation was started $24 \mathrm{~h}$ after his dose of intravenous t-PA.

MRI and MRA of the head and neck was done the following day, showing a stable appearance to the dissection and a right superior division MCA territory stroke (Figure 6). He was discharged from the hospital 5 days later with an NIHSS of 1 . Six weeks later, his 


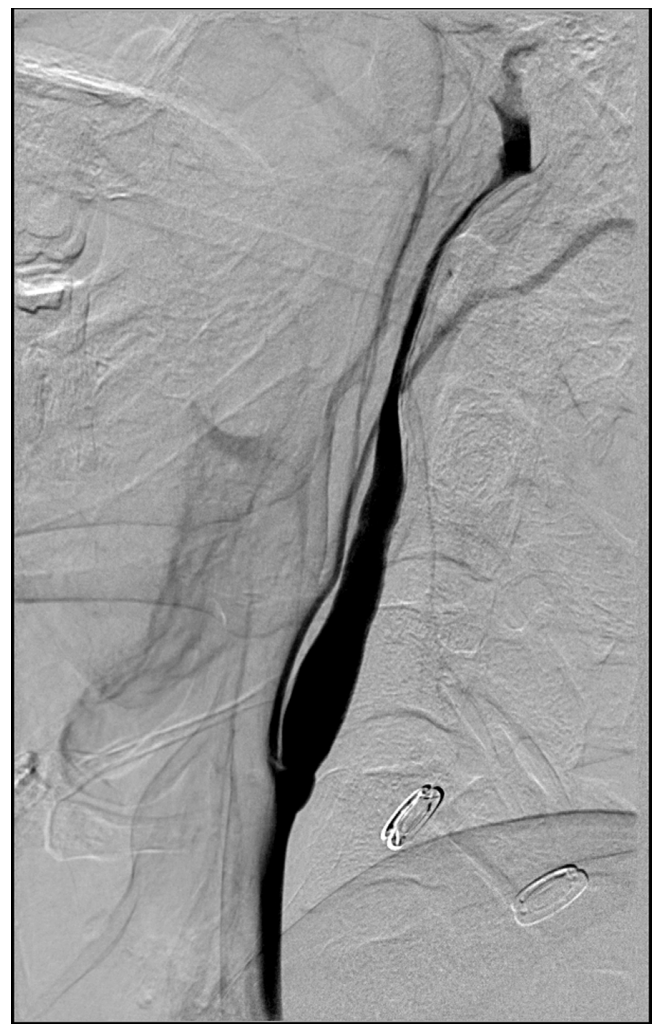

FIGURE 1 | Right lateral common carotid digital subtraction angiography cervical view showing an intimal flap and smooth tapered cervical carotid dissection extending up to the cervical/petrous junction.

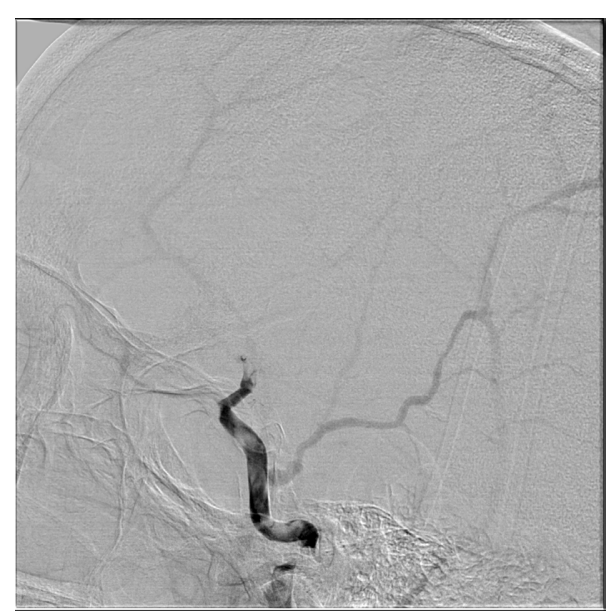

FIGURE 2 | Right lateral common carotid digital subtraction angiography cerebral view showing markedly delayed anterograde flow in the internal carotid artery with a supraclinoid occlusion and flow defects in petrous and cavernous section of carotid artery.

NIHSS score remained 1 with slight word finding difficulties and MRA showed persistence of the right cervical/petrous dissection. He was maintained on anticoagulation for 6 months and on 12 month followup, his NIHSS score was 0 and he was back to work in construction.

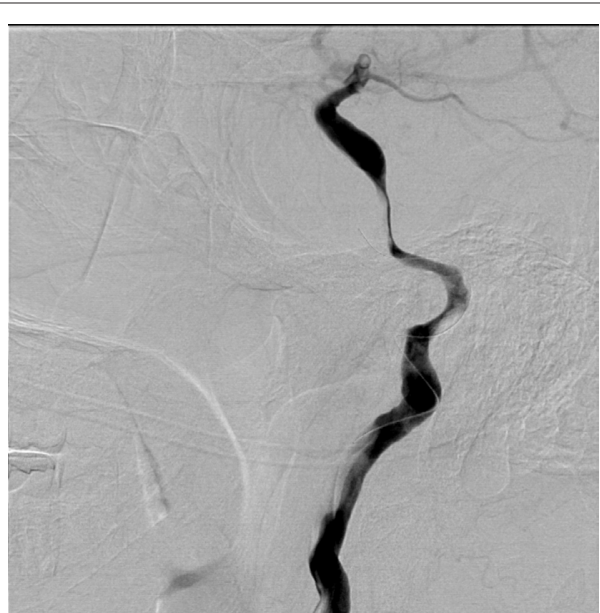

FIGURE 3 | Right lateral common carotid digital subtraction angiography cervical view showing microguidewire in the dissected segment of cervical carotid artery, worsening the underlying pathology.

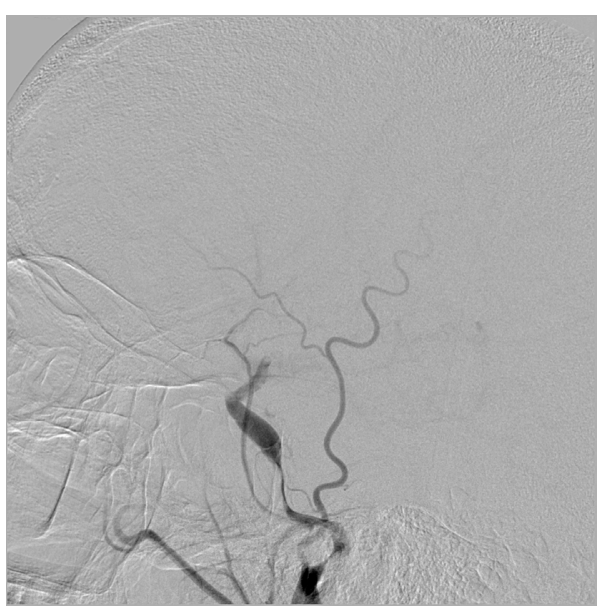

FIGURE 4 | Right lateral common carotid digital subtraction angiography cerebral view showing a near occlusive intimal flap at the cervical petrous section.

\section{DISCUSSION}

In patients younger than age 45 , arterial dissection is a leading cause of stroke (Lisovoski and Rousseaux, 1991; Schievink et al., 1994). Carotid artery intimal tears leading to dissection usually occur a few centimeters distal to the carotid bulb, at the $\mathrm{C} 2-\mathrm{C} 3$ level, and the dissection usually extends distally until the carotid artery enters the foramen lacerum. The typical angiographic finding is a relatively smooth or slightly irregular tapered mid-cervical narrowing (Provenzale, 1995). Carotid subintimal dissections are dynamic lesions, and depending on the size, can cause just minor luminal narrowing, or complete occlusion. As a result, symptoms are variable and can range from cranial nerve compromise, to a Horner's syndrome, to ischemia in vascular territories distal to the lesion. Most ischemic symptoms occur within 


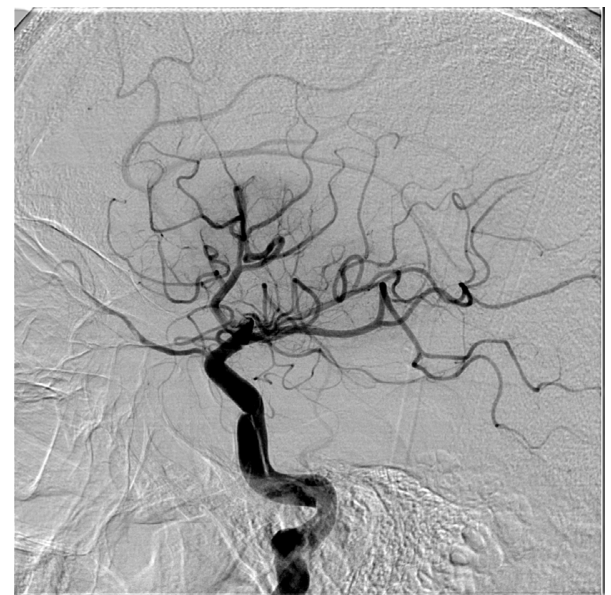

FIGURE 5 |After proximal flow arrest and aggressive proximal guiding catheter aspiration, TIMI 3 flow in the internal carotid is restored. The dissection is now seen to extend into the petrous section, but is not flow limiting.

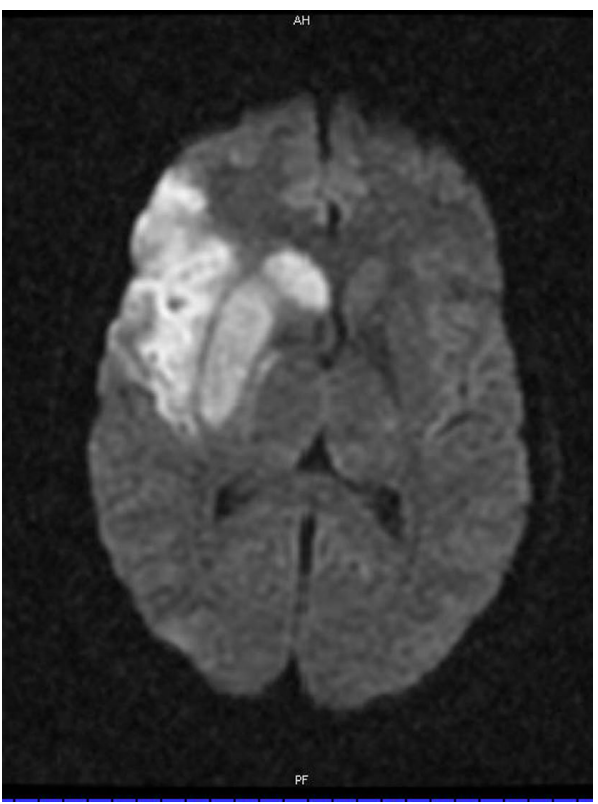

FIGURE 6 | Diffusion weighted MRI at 24 hours showing increased signal in a superior division middle cerebral artery branch territory.

1 week of the onset of pain, as was the time course in this patient (Biousse et al., 1995). Most infarctions are territorial, rather than borderzone, indicating an embolic rather than hemodynamic etiology (Provenzale, 1995).

Increasing familiarity with endovascular revascularization devices allows us to tailor our approach based on our attempts at understanding stroke pathophysiology. Mechanical revascularization of severe carotid dissections using microcatheters and wires may worsen the underlying pathology. Dissections are delicate, dynamic lesions that are not easily navigated around using exist- ing biplane angiographic imaging. With an occlusive distal clot, roadmap imaging is often of poor image quality because arterial washout is delayed. Furthermore, in the acute phase, anterograde blood flow is steadily applying a force underneath the intimal flap, predisposing it to worsen. With conscious sedation rather than general anesthesia being more commonly used, patient movement during the procedure is not only to be expected, but adds additional challenge to not only discerning the false from the true lumen, but also navigating a microcatheter past the lesion (Abou-Chebl et al., 2010).

Pharmacologic, intra-arterial thrombolysis may also be problematic in the setting of acute stroke caused by arterial dissection. Published small case series of intra-arterial thrombolysis for acute stroke due to arterial dissection have not shown significant rates of rupture of dissected vessels, cervical or subarachnoid hemorrhage, intracranial hemorrhage, or peri-interventional arterial embolism (Arnold et al., 2000). However, chemical thrombolytics such as urokinase or t-PA carry the theoretical risk of extending the arterial wall hematoma, independent of the microcatheter inadvertently widening the false lumen.

Another approach to this complicated scenario is to treat and stabilize the cervical carotid or vertebral dissection first, thereby facilitating access to the distal intracranial thromboembolus. Several reports have described cervical carotid angioplasty and stent placement as treatment option for arterial dissection (Abboud et al., 2005). This approach may have a role in not only acute recanalization, but also for secondary stroke prevention, permitting immediate and durable recanalization of the artery but associated with use of dual antiplatelet therapy instead of anticoagulation. A few technical drawbacks exist. First, the passage of a wire or embolic protection device and stent delivery catheter past the dissection may, again, widen the false lumen. Secondly, distal embolic protection devices may not be as easily used as the dissected segment often extends to the cervical/ petrous section. Another concern for stent placement in a dissected artery is the weakened structural integrity of the vessel wall, unlike that of an atherosclerotic vessel wall, which is the pathology for which this device is designed to treat. The vessel may be undergo additional injury with either balloon angioplasty and/or stent placement. Lastly, dissected segments, unlike atherosclerotic lesions for which currently available stents are designed for, are often much longer, and difficult to accurately determine the proximal and distal margins. Accurately reopposing the stent along the entire dissected segment may require multiple overlapping stents.

Proximal aspiration through an 8F guiding catheter has been previously described in case reports to treat carotid bulb occlusion (Xu et al., 2005). Technical feasibility was demonstrated with a 50 -cc syringe with good clinical and angiographic results. However, without a proximal balloon and the ability to induce flow arrest, they were limited in their ability to mechanically disrupt the clot for fear of distal emboli. In both cases however, pretreatment angiography suggested a significant clot burden that was amenable to aggressive proximal aspiration.

The concept behind proximal flow control to support retrieval of thrombus was published prior to the release of the Merci Retrieval system in 2004. It was based on the concept 
that anterograde flow maintained the clot in place like a cork, and reducing this force would then facilitate its removal (Mayer et al., 2002).

In our case, precious time was spent gaining access and attempting to retrieve the distal clot with microcatheters and wires. Furthermore, at one point, the dissection was worsened as a result of these maneuvers. We were most successful with recanalization when we applied proximal flow arrest followed by aggressive proximal aspiration through the $8 \mathrm{~F}$ guide catheter situated below the dissection. Fortunately, placing the end of the guide catheter at the level of the carotid bulb appears safe as most carotid dissections occur just distal to the bulb. Proximal flow arrest with proximal aspiration appears to be a safe and effective adjunctive technique for these lesions. Some may argue that aspiration alone may have accounted for the recanalization. As such, the dissection is not mechanically disturbed by attempts at advancing a microcatheter past it. Furthermore, flow reversal is associated with a retrograde force on the intimal flap that may facilitate re-apposition of the normal arterial layers.

\section{REFERENCES}

Abboud, H., Houdart, E., Meseguer, E., and Amarenco, P. (2005). Stent assisted endovascular thrombolysis of internal carotid artery dissection. J. Neurol. Neurosurg. Psychiatry 76, 292-293.

Abou-Chebl, A., Lin, R., Hussain, M., Jovin, T. G., Levy, E. I., Liebeskind, D. S., Yoo, A. J., Hsu, D. P., Rymer, M. M., Tayal, A. H., Zaidat, O. O., Natarajan, S. K., Nogueira, R. G., Nanda,A., Tian, M., Hao Q., Kalia, J. S., Nguyen, T. N., Chen, M., and Gupta, R. (2010). Conscious sedation versus general anesthesia during endovascular therapy for acute anterior circulation stroke: preliminary results from a retrospective multi-center study. Stroke 41, 1175-1179.
Arnold, M., Nedeltchev, K., Sturzenegger, M., Schroth, G., Loher, T. J., Stepper, F., Remonda,L., Bassetti, C., and Mattle, $\mathrm{H}$. P. (2000). Thrombolysis in patients with acute stroke caused by cervical artery dissection. Neurology 54, 2159-2161.

Biousse, V., D’Anglejean-Chatillon, J., Touboul, P.-J., Amarenco, P., and Bousser, M. G. (1995). Time course of symptoms in extracranial carotid artery dissections. Stroke 26, 235.

Lisovoski, F., and Rousseaux, P. (1991) Cerebral infarction in young people. A study of 148 patients with early cerebral angiography. J. Neurol. Neurosurg. Psychiatry 54, 576-579.

Mayer, T. E., Hamann, G. F., and Brueckmann, H. J. (2002). Treatment of basilar artery embolism with a mechanical extraction device:

An analogous situation occurs with an occlusive lesion that extends from the distal cervical carotid to the supraclinoid carotid with intact interhemispheric flow via the anterior communicating artery. Again, passing any type of device through or within the distal aspect of the clot runs the risk of compromising the functioning circle of Willis collaterals. A proximal-to-the-clot approach may have an advantage of preserving the circle of Willis collaterals.

\section{CONCLUSION}

The use of acute endovascular stroke therapy for a large artery ischemic stroke secondary to arterial dissections involves unique modifications to the conventional procedural protocol. This case review highlights the importance of careful navigation of any microcatheters, wires, and devices across the arterial dissection, as well as the potential utility of aggressive aspiration via a large bore guiding catheter proximal to the dissection.

Ethics Approval Statement: This study was approved by Rush University Medical Center, Chicago, IL, USA, IRB\#07110702.

necessity of flow reversal. Stroke 33, 2232-2235.

Provenzale, J. W. (1995). Dissection of the internal carotid and vertebral arteries: imaging features. AJR Am. J. Roentgenol. 165, 1099-1104.

Schievink, W.I., Mokri, B., and Jackers, G. (1994). Spontaneous dissection of cervicocephalic arteries in childhood and adolescence. Neurology 44, 1607.

Xu, G. F., Sub, D. C., Choi, C. G., Kim, J. K., Kim, W., Kim, S. J., and Kim, J. (2005). Aspiration thrombectomy of acute complete carotid bulb occlusion. J. Vasc. Interv. Radiol. 16, 539-542.

Conflict of Interest Statement: The author declares that the research was conducted in the absence of any commercial or financial relationships that could be construed as a potential conflict of interest.

Received: 25 June 2010; paper pending published:08 October 2010; accepted:08 October 2010; published online: 04 November 2010. Citation: Chen M (2010) Mechanical recanalization of acute carotid terminus occlusion from traumatic arterial dissection. Front Neur. 1:123. doi: 10.3389/fneur.2010.00123 This article was submitted to Frontiers in Endovascular and Interventional Neurology, a specialty of Frontiers in Neurology. Copyright (c) 2010 Chen. This is an openaccess article subject to an exclusive license agreement between the authors and the Frontiers Research Foundation, which permits unrestricted use, distribution, and reproduction in any medium, provided the original authors and source are credited. 